TAIWANESE JOURNAL OF MATHEMATICS

Vol. 17, No. 1, pp. 207-219, February 2013

DOI: $10.11650 /$ tjm.17.2013.1909

This paper is available online at http://journal.taiwanmathsoc.org.tw

\title{
DISTANCE THREE LABELINGS FOR DIRECT PRODUCTS OF THREE COMPLETE GRAPHS
}

\author{
Byeong Moon Kim, Byung Chul Song and Woonjae Hwang*
}

\begin{abstract}
The distance 3 labeling number $\lambda_{G}\left(j_{0}, j_{1}, j_{2}\right)$ for a graph $G=(V, E)$ is the smallest integer $\alpha$ such that there is a function $f: V \rightarrow[0, \alpha]$, satisfying $|f(u)-f(v)| \geq j_{\delta-1}$ for any pair of vertices $u, v$ of distance $\delta \leq 3$. In this paper, we determine the distance 3 labeling number $\lambda_{G}(j, k, 1)$ for the direct product $G=K_{n} \times K_{m} \times K_{2}(n \geq m \geq 3)$ of 3 complete graphs under various conditions on $j$ and $k$. As a consequence, we have the radio number $\operatorname{mn}(G)=2 m n-1$.
\end{abstract}

\section{INTRODUCTION}

The channel assignment problem introduced by Hale [9] is the motivation for the various labeling problems in graphs. For a graph $G=(V, E)$ and nonnegative integers $\alpha, j_{0}, j_{1}, \cdots, j_{d-1}$, an $L\left(j_{0}, j_{1}, \cdots, j_{d-1}\right)$-labeling (or distance d-labeling) is an integer valued function $f: V \rightarrow[0, \alpha]$ such that for $u, v \in V$ with $\delta=$ $\operatorname{dist}(u, v)$, the labeling condition $|f(u)-f(v)| \geq j_{\delta-1}$ is satisfied. The labeling number $\lambda_{G}\left(j_{0}, j_{1}, \cdots, j_{d-1}\right)$ for $G$ is the smallest integer $\alpha$ such that there is an $L\left(j_{0}, j_{1}, \cdots, j_{d-1}\right)$-labeling $f: V \rightarrow[0, \alpha]$. The distance 2 labeling has been studied the most. In particular, the labeling number $\lambda_{G}(2,1)$ is referred to as the $\lambda$-number for $G$, and is denoted by $\lambda(G)$. After the work by Griggs and Yeh [8], special attention has been paid to the $\lambda$-number of graphs.

The radio labeling for $G$ is the $L(d, d-1, \cdots, 1)$-labeling $f$, where $d=\operatorname{diam}(G)$. In this case, the labeling condition between two vertices $u$ and $v$ is $|f(u)-f(v)| \geq$ $d-\delta+1$, where $\delta=\operatorname{dist}(u, v)$. The radio number for $G$, denoted by $\operatorname{rn}(G)$, is the minimum span of radio labelings for $G$. If a graph has diameter 2, then $\lambda(G)=\operatorname{rn}(G)$.

Liu and Zhu [16] completely determined the radio numbers for paths and cycles. Liu [13] treated the radio number for trees. Liu and Xie [17] determined the radio

Received April 12, 2012, accepted June 27, 2012.

Communicated by Gerard Jennhwa Chang.

2010 Mathematics Subject Classification: 05C78, 05C12, 05 C76.

Key words and phrases: Distance three labeling, Radio number, Direct products, Complete graphs.

This work was supported by Korea University grant.

*Corresponding author. 
number for the squares of paths and squares of some cycles. Recently Li, Mak and Zhou [15] found the optimal radio labelings of complete $m$-ary trees. There are some results on the distance 3 labelings for graphs. In particular, $\lambda_{G}(1,1,1)$ and $\lambda_{G}(2,1,1)$ have been computed when $G$ is a path, cycle, grid, complete binary tree, or cube $[1,2,3,4,7,13,16,19]$. Recently, Chia et al. [6] gave the upper bound of $\lambda_{G}(3,2,1)$ when $G$ is a general graph, and when $G$ is a tree. They also computed $\lambda_{G}(3,2,1)$ for some classes of $G$. In addition, $L(h, 1,1)$-labelings of trees and outplanar graphs are studied recently in $[5,12]$.

The direct product of $n$ graphs $G_{i}=\left(V_{G_{i}}, E_{G_{i}}\right),(i=1,2, \cdots, n)$ is the graph $G=$ $G_{1} \times G_{2} \times \cdots \times G_{n}$ that the vertex set is $V_{G_{1}} \times V_{G_{2}} \times \cdots \times V_{G_{n}}$ and $\left[\left(u_{1}, u_{2}, \cdots, u_{n}\right)\right.$, $\left.\left(v_{1}, v_{2}, \cdots, v_{n}\right)\right]$ belongs to the edge set if $\left(u_{i}, v_{i}\right) \in E_{G_{i}}$ for all $i$. It is easy to see that $G=K_{n} \times K_{m} \times K_{l}(n \geq m \geq l)$ is of diameter 2 when $l \geq 3$, and of diameter 3 when $l=2$ and $m \geq 3$. Further, $G=K_{n} \times K_{m} \times K_{l}$ is disconnected when $l=m=2$, in which case, $G$ is isomorphic to two copies of $K_{n} \times K_{2}$. Haque and Jha [10] and Lam et al. [14] studied the $L(j, k)$-labeling number for the multiple direct product of complete graphs.

In this paper, we show that when $G=K_{n} \times K_{m} \times K_{2}, n \geq m \geq 3$, the distance 3 labeling number $\lambda_{G}(j, k, 1)$ for $G$ satisfies

$$
\lambda_{G}(j, k, 1)= \begin{cases}2 m n-1 & \text { if } k=1 \text { and } 1 \leq j \leq n+2 . \\ (m n-1) k+1 & \text { if } k \geq 2 \text { and } k \leq j \leq 2 k-1 . \\ (m n-1) k+m & \text { if } k \geq 2 \text { and } j=2 k . \\ (m-1) j+(m n-2 m+1) k+1 & \text { if } k \geq 2 \text { and } 2 k+1 \leq j \leq n k .\end{cases}
$$

As a consequence, we prove that the radio number $\operatorname{rn}(G)$ for $G$ is $2 m n-1$.

\section{Preliminaries and Some Lemmas}

Henceforth, we assume that $n \geq m \geq 3, j \geq k \geq 2, G=K_{n} \times K_{m} \times K_{2}=(V, E)$, and $f: V \rightarrow[0, \alpha]$ is an $L(j, k, 1)$-labeling. The vertices $x_{1}, x_{2}, \cdots, x_{p}$ are on the same row, same column, or same floor if they have the same first, second, or third components, respectively. Two vertices $u$ and $v$ on the same floor are said to be set oblique if they are on different rows and columns. Since the distance between two vertices on the same floor is 2 , if $x_{1}, x_{2}, \cdots, x_{p}$ are on the same floor, $f\left(x_{1}\right)<$ $f\left(x_{2}\right)<\cdots<f\left(x_{p}\right)$ and $1 \leq t<s \leq p$, and then, we have $f\left(x_{s}\right)-f\left(x_{t}\right) \geq k(t-s)$. Let $V_{i}=\left\{\left(u_{0}, u_{1}, u_{2}\right) \mid u_{2}=i\right\}$ for $i=0,1$ be the two floors of $V$.

Lemma 1. Let $x_{1}, x_{2}, x_{3} \in V_{0}$ and $y_{1}, y_{2} \in V_{1}$. If $x_{1}$ and $x_{2}$ are set oblique, then there exist $x \in\left\{x_{1}, x_{2}, x_{3}\right\}$ and $y \in\left\{y_{1}, y_{2}\right\}$ such that $x$ and $y$ are adjacent.

Proof. Suppose that no element of $x_{1}$ and $x_{2}$ is adjacent to any of $y_{1}$ and $y_{2}$. Since $x_{1}=\left(u_{0}, u_{1}, 0\right)$ and $x_{2}=\left(v_{0}, v_{1}, 0\right)$ are set oblique, $u_{0} \neq v_{0}$ and $u_{1} \neq v_{1}$. 
Since the two non adjacent vertices share some components, we have only two vertices of $V_{1}-\left(u_{0}, v_{1}, 1\right)$ and $\left(v_{0}, u_{1}, 1\right)$ - that are adjacent to neither $x_{1}$ nor $x_{2}$. As such, there exist $y_{1}$ and $y_{2}$ that are set oblique. Similarly, $x_{1}$ and $x_{2}$ are the only vertices of $V_{0}$ that are adjacent to neither $y_{1}$ nor $y_{2}$. Hence, $x_{3}$ is adjacent to $y_{1}$ or $y_{2}$.

Lemma 2. If $x_{1}, x_{2}, \cdots, x_{n+1} \in V_{i}$ for some $i=0,1$, then there exists a pair of vertices that are set oblique among $x_{1}, x_{2}, \cdots, x_{n+1}$.

Proof. Suppose there exists no pair of vertices among vertices $x_{1}, x_{2}, \cdots, x_{n+1}$ that are set oblique. Since $x_{1}$ and $x_{2}$ are not set oblique, they are on the same row or the same column. Since $n \geq m$, we can assume that they are on the same row. Since there are $n+1$ vertices on one floor, there exists $x_{i}$ that is not on the same row as $x_{1}$. Since $x_{1}$ and $x_{2}$ are not on the same column, $x_{i}$ is not on the same column as $x_{1}$ or $x_{2}$. As such either $x_{i}$ and $x_{1}$ or $x_{i}$ and $x_{2}$ are set oblique.

Lemma 3. For $p \geq 3$, let $x_{1}, x_{2}, \cdots, x_{p} \in V_{i}$ for some $i=0,1$. If there exists a pair of vertices among $x_{1}, x_{2}, \cdots, x_{p}$ that are set oblique, then there is an integer $s(1 \leq s \leq p-2)$ such that $\left\{x_{s}, x_{s+1}, x_{s+2}\right\}$ contains a pair of vertices set oblique.

Proof. Among the pair $(r, t)$, where $x_{r}$ and $x_{t}$ are set oblique, choose $\left(r_{0}, t_{0}\right)$ such that $|t-r|$ takes the minimum. We can assume that $r_{0}<t_{0}$. It suffices to show that $t_{0}-r_{0} \leq 2$. On the contrary, assume that $t_{0}-r_{0} \geq 3$. If $x_{r_{0}}$ and $x_{r_{0}+1}$ are not set oblique, then they are on the same row or the same column. We can assume that they are on the same row. Since $x_{r_{0}+2}$ and $x_{t_{0}}$ are not set oblique, they are on the same row or the same column. If they are on the same row, then $x_{r_{0}}$ and $x_{r_{0}+2}$ are on the same column. Since $x_{r_{0}}$ and $x_{r_{0}+1}$ are on the same row, and $x_{r_{0}+2}$ and $x_{t_{0}}$ are on the same row, $x_{r_{0}+1}$ and $x_{r_{0}+2}$ are on distinct rows. Since $x_{r_{0}}$ and $x_{r_{0}+1}$ are on distinct columns, $x_{r_{0}+1}$ and $x_{r_{0}+2}$ are on distinct columns. As such, $x_{r_{0}+1}$ and $x_{r_{0}+2}$ are set oblique. This is a contradiction. If $x_{r_{0}+2}$ and $x_{t_{0}}$ are on the same column, since $x_{r_{0}}$ and $x_{r_{0}+2}$ are not set oblique, then they are on the same row. Then, $x_{r_{0}+1}=x_{r_{0}+2}$. This is also a contradiction. Hence, $t_{0}-r_{0} \leq 2$.

For $S \subset V$ and labeling $f$, we define the span $\operatorname{span}(f: S)$ of $f$ on $S$ as the maximum of $|f(u)-f(v)|$ for $u, v \in S$. The span $\operatorname{span}(f)$ of $f$ is $\operatorname{span}(f: V)$. Let $|S|=2 n+1, S \cap V_{0}=\left\{x_{1}, x_{2}, \cdots, x_{p}\right\}$ and $S \cap V_{1}=\left\{y_{1}, y_{2}, \cdots, y_{q}\right\}$ with $f\left(x_{1}\right)<f\left(x_{2}\right)<\cdots<f\left(x_{p}\right)$ and $f\left(y_{1}\right)<f\left(y_{2}\right)<\cdots<f\left(y_{q}\right)$. Without loss of generality, we can assume that $p \geq q$, and hence, we have $p \geq n+1$. From Lemmas 2 and 3 , there exists $s(1 \leq s \leq p-2)$ such that $\left\{x_{s}, x_{s+1}, x_{s+2}\right\}$ contains a pair of vertices that are set oblique. Then, $p+q=2 n+1$ and we have the following propositions.

Proposition 1. If $\left\{x_{s}, x_{s+1}, x_{s+2}\right\}$ contains a pair of vertices that are set oblique, $f\left(y_{2}\right)<f\left(x_{s+2}\right)$, and $f\left(x_{s}\right)<f\left(y_{q-1}\right)$, then $\operatorname{span}(f: S) \geq j+(n-2) k$. 
Proof. Since $p-3 \geq n-2$ and $\left\lceil\frac{p+q-6}{2}\right\rceil \geq\left\lceil\frac{2 n+1-6}{2}\right\rceil=n-2$, it is enough to show that $\operatorname{span}(f: S) \geq \min \left\{j+(p-3) k, j+\left\lceil\frac{p+q-6}{2}\right\rceil k\right\}$.

Since $f\left(y_{2}\right)<f\left(x_{s+2}\right)$ and $f\left(x_{s}\right)<f\left(y_{q-1}\right)$, there exist $\alpha, \beta$ such that $\alpha$ is the smallest number $h$ such that $f\left(y_{h}\right)>f\left(x_{s}\right)$ and $\beta$ is the largest number $h$ such that $f\left(y_{h}\right)<f\left(x_{s+2}\right)$. If $\alpha \geq \beta+2$, then $f\left(x_{s+2}\right)<f\left(y_{\beta+1}\right) \leq f\left(y_{\alpha-1}\right)<f\left(x_{s}\right)$. This is a contradiction. Hence, $\alpha \leq \beta+1$. Since $(s+q-\alpha+1)+(\beta+p-s-1)=$ $p+q-\alpha+\beta \geq p+q-1=2 n$, there are three possible cases:

Case 1: $s+q-\alpha+1 \geq\left\lceil\frac{p+q}{2}\right\rceil=n+1$.

Case 2: $\beta+p-s-1 \geq\left\lceil\frac{p+q}{2}\right\rceil=n+1$.

Case 3: $s+q-\alpha+1=\beta+p-s-1=n$.

Case 1. $s+q-\alpha+1 \geq\left\lceil\frac{p+q}{2}\right\rceil$.

From Lemma 1, some $x \in\left\{x_{s}, x_{s+1}, x_{s+2}\right\}$ is adjacent to some $y \in\left\{y_{\alpha}, y_{\alpha+1}\right\}$. If $f\left(y_{\alpha+1}\right)>f\left(x_{s+2}\right)$, then $f\left(y_{\alpha+1}\right)-f\left(x_{s}\right) \geq|f(x)-f(y)| \geq j$. As such,

$\operatorname{span}(f: S) \geq f\left(y_{q}\right)-f\left(x_{1}\right)$

$$
\begin{aligned}
& =\left(f\left(y_{q}\right)-f\left(y_{\alpha+1}\right)\right)+\left(f\left(y_{\alpha+1}\right)-f\left(x_{s}\right)\right)+\left(f\left(x_{s}\right)-f\left(x_{1}\right)\right) \\
& \geq(q-\alpha-1) k+j+(s-1) k=j+(s+q-\alpha-2) k \\
& \geq j+(n-2) k .
\end{aligned}
$$

If $f\left(x_{s+2}\right)>f\left(y_{\alpha+1}\right)$, then $f\left(x_{s+2}\right)-f\left(x_{s}\right) \geq|f(x)-f(y)| \geq j$. Thus,

$$
\begin{aligned}
\operatorname{span}(f: S) & \geq f\left(x_{p}\right)-f\left(x_{1}\right) \\
& =\left(f\left(x_{p}\right)-f\left(x_{s+2}\right)\right)+\left(f\left(x_{s+2}\right)-f\left(x_{s}\right)\right)+\left(f\left(x_{s}\right)-f\left(x_{1}\right)\right) \\
& \geq(p-s-2) k+j+(s-1) k=j+(p-3) k \geq j+(n-2) k .
\end{aligned}
$$

Case 2. $\beta+p-s-1 \geq\left\lceil\frac{p+q}{2}\right\rceil=n+1$.

From Lemma 1, some $x \in\left\{x_{s}, x_{s+1}, x_{s+2}\right\}$ is adjacent to some $y \in\left\{y_{\beta-1}, y_{\beta}\right\}$. If $f\left(y_{\beta-1}\right)<f\left(x_{s}\right)$, then $f\left(x_{s+2}\right)-f\left(y_{\beta-1}\right) \geq|f(x)-f(y)| \geq j$. Hence,

$$
\begin{aligned}
\operatorname{span}(f: S) & \geq f\left(x_{p}\right)-f\left(y_{1}\right) \\
& =\left(f\left(x_{p}\right)-f\left(x_{s+2}\right)\right)+\left(f\left(x_{s+2}\right)-f\left(y_{\beta-1}\right)\right)+\left(f\left(y_{\beta-1}\right)-f\left(y_{1}\right)\right) \\
& \geq(p-s-2) k+j+(\beta-2) k=j+(p-s+\beta-4) k \\
& \geq j+\left\lceil\frac{p+q-6}{2}\right\rceil k \\
& \geq j+(n-2) k .
\end{aligned}
$$

If $f\left(y_{\beta-1}\right)>f\left(x_{s}\right)$, then $f\left(x_{s+2}\right)-f\left(x_{s}\right) \geq|f(x)-f(y)| \geq j$. Therefore, $\operatorname{span}(f: S) \geq f\left(x_{p}\right)-f\left(x_{1}\right)$

$$
\begin{aligned}
& =\left(f\left(x_{p}\right)-f\left(x_{s+2}\right)\right)+\left(f\left(x_{s+2}\right)-f\left(x_{s}\right)\right)+\left(f\left(x_{s}\right)-f\left(x_{1}\right)\right) \\
& \geq(p-s-2) k+j+(s-1) k=j+(p-3) k \\
& \geq j+(n-2) k .
\end{aligned}
$$


Case 3. $s+q-\alpha+1=\beta+p-s-1=\frac{p+q-1}{2}=n$.

From Lemma 1 , some $x \in\left\{x_{s}, x_{s+1}, x_{s+2}\right\}$ is adjacent to some $y \in\left\{y_{\alpha}, y_{\beta}\right\}$. Since $\alpha=\beta+1, f\left(y_{\beta}\right)=f\left(y_{\alpha-1}\right)<f\left(x_{s}\right)$ and $f\left(y_{\alpha}\right)=f\left(y_{\beta+1}\right)>f\left(x_{s+2}\right)$. If $y=y_{\alpha}$, $f\left(y_{\alpha}\right)-f\left(x_{s}\right) \geq|f(y)-f(x)| \geq j$. As such,

$$
\begin{aligned}
\operatorname{span}(f: S) & \geq f\left(y_{q}\right)-f\left(x_{1}\right) \\
& =\left(f\left(y_{q}\right)-f\left(y_{\alpha}\right)\right)+\left(f\left(y_{\alpha}\right)-f\left(x_{s}\right)\right)+\left(f\left(x_{s}\right)-f\left(x_{1}\right)\right) \\
& \geq(q-\alpha) k+j+(s-1) k=j+(q+s-\alpha-1) k \\
& =j+(n-2) k .
\end{aligned}
$$

If $y=y_{\beta}$, then $f\left(x_{s+2}\right)-f\left(y_{\beta}\right) \geq|f(y)-f(x)| \geq j$. Thus,

$$
\begin{aligned}
\operatorname{span}(f: S) & \geq f\left(x_{p}\right)-f\left(y_{1}\right) \\
& =\left(f\left(x_{p}\right)-f\left(x_{s+2}\right)\right)+\left(f\left(x_{s+2}\right)-f\left(y_{\beta}\right)\right)+\left(f\left(y_{\beta}\right)-f\left(y_{1}\right)\right) \\
& \geq(p-s-2) k+j+(\beta-1) k=j+(p+\beta-s-3) k \\
& =j+(n-2) k .
\end{aligned}
$$

Proposition 2. Let $|S|=p+q=2 n+1$ and $j \geq 2 k$.

(1) If $q \leq 2$, then $\operatorname{span}(f: S) \geq(2 n-2) k$.

(2) If $q \geq 3$, then $\operatorname{span}(f: S) \geq j+(n-2) k$.

(3) If $j=2 k$, then $\operatorname{span}(f: S) \geq n k+1$.

\section{Proof.}

(1) In this case, $p \geq 2 n-1$, and we have

$$
\begin{aligned}
\operatorname{span}(f: S) & \geq f\left(x_{p}\right)-f\left(x_{1}\right) \\
& \geq(p-1) k \geq(2 n-1-1) k=(2 n-2) k .
\end{aligned}
$$

(2) From Lemmas 2 and 3, there exists $s(1 \leq s \leq p-2)$ such that $\left\{x_{s}, x_{s+1}, x_{s+2}\right\}$ contains a pair of vertices that are set oblique. Let $s_{1}<s_{2}<\cdots<s_{h}$ be all $s$ such that $\left\{x_{s}, x_{s+1}, x_{s+2}\right\}$ contains a pair of vertices that are set oblique. From Lemmas 2 and $3, s_{i+1}-s_{i} \leq n-1$ for all $i=1,2, \cdots, h-1$.

Case 1. $f\left(y_{q-1}\right)<f\left(x_{s_{1}}\right)$. If $\alpha=s_{1}$, from Lemma $2, \alpha \leq n-1$. If $y_{q-1}$ is adjacent to some $x_{\alpha}, x_{\alpha+1}$, and $x_{\alpha+2}$, since $f\left(x_{\alpha+2}\right)-f\left(y_{q-1}\right) \geq j$, we have

$$
\begin{aligned}
\operatorname{span}(f: S) & \geq f\left(x_{p}\right)-f\left(y_{1}\right) \\
& =\left(f\left(x_{p}\right)-f\left(x_{\alpha+2}\right)\right)+\left(f\left(x_{\alpha+2}\right)-f\left(y_{q-1}\right)\right)+\left(f\left(y_{q-1}\right)-f\left(y_{1}\right)\right) \\
& \geq(p-\alpha-2) k+j+(q-2) k=j+(p+q-\alpha-4) k \\
& \geq j+(2 n+1-(n-1)-4) k=j+(n-2) k .
\end{aligned}
$$


If $y_{q-1}$ is adjacent to no $x_{\alpha}, x_{\alpha+1}$, and $x_{\alpha+2}$, then from Lemma $1, y_{q}$ is adjacent to some $x_{\alpha}, x_{\alpha+1}$, and $x_{\alpha+2}$. Moreover, from Lemma 1, $y_{q-2}$ is adjacent to some $x_{\alpha}, x_{\alpha+1}$, and $x_{\alpha+2}$. If $f\left(y_{q}\right)<f\left(x_{\alpha}\right)$, since $f\left(x_{\alpha+2}\right)-f\left(y_{q}\right) \geq f(x)-f\left(y_{q}\right) \geq$ $j$, we have

$$
\begin{aligned}
\operatorname{span}(f: S) & \geq f\left(x_{p}\right)-f\left(y_{1}\right) \\
& =\left(f\left(x_{p}\right)-f\left(x_{\alpha+2}\right)\right)+\left(f\left(x_{\alpha+2}\right)-f\left(y_{q}\right)\right)+\left(f\left(y_{q}\right)-f\left(y_{1}\right)\right) \\
& \geq j+(p+q-\alpha-3) k \geq j+(n-2) k .
\end{aligned}
$$

If $f\left(y_{q}\right)>f\left(x_{\alpha}\right)$ and $\alpha \leq n-2$, then since $y_{q-2}$ is adjacent to some $x_{\alpha}, x_{\alpha+1}$, and $x_{\alpha+2}$, and since $f\left(x_{\alpha+2}\right)-f\left(y_{q-2}\right) \geq f(x)-f\left(y_{q-2}\right) \geq j$, we have

$$
\begin{aligned}
\operatorname{span}(f: S) & \geq f\left(x_{p}\right)-f\left(y_{1}\right) \\
& =\left(f\left(x_{p}\right)-f\left(x_{\alpha+2}\right)\right)+\left(f\left(x_{\alpha+2}\right)-f\left(y_{q-2}\right)\right)+\left(f\left(y_{q-2}\right)-f\left(y_{1}\right)\right) \\
& \geq j+(p+q-\alpha-5) k \geq j+(n-2) k .
\end{aligned}
$$

If $f\left(y_{q}\right)>f\left(x_{\alpha}\right)$ and $\alpha=n-1$, since $x$ is adjacent to $y_{q},\left|f(x)-f\left(y_{q}\right)\right| \geq j$. If $f(x)>f\left(y_{q}\right)$, then $f\left(x_{\alpha+2}\right)-f\left(x_{\alpha}\right) \geq f(x)-f(y) \geq j$. If $f(x)<f\left(y_{q}\right)$, then $f\left(y_{q}\right)-f\left(x_{\alpha}\right) \geq f\left(y_{q}\right)-f(x) \geq j$. As such,

$$
\begin{aligned}
\operatorname{span}(f: S) & \geq \max \left\{f\left(x_{\alpha+2}\right)-f\left(x_{1}\right), f\left(y_{q}\right)-f\left(x_{1}\right)\right\} \\
& \geq \max \left\{f\left(x_{\alpha+2}\right)-f\left(x_{\alpha}\right), f\left(y_{q}\right)-f\left(x_{\alpha}\right)\right\}+\left(f\left(x_{\alpha}\right)-f\left(x_{1}\right)\right) \\
& \geq j+(\alpha-1) k \geq j+(n-2) k .
\end{aligned}
$$

Case 2. $f\left(y_{q-1}\right)>f\left(x_{s_{1}}\right)$. Let $i$ be the largest $t$ such that $f\left(x_{s_{t}}\right)<f\left(y_{q-1}\right)$. If $f\left(x_{s_{i}+2}\right)>f\left(y_{2}\right)$, then from Proposition 1, $\operatorname{span}(f: S) \geq j+(n-2) k$. Therefore, we can assume that $f\left(x_{s_{i}+2}\right)<f\left(y_{2}\right)$.

If $i=h$, then $f\left(x_{s_{h}+2}\right)<f\left(y_{2}\right)$. As in Case 1, we can prove that $\operatorname{span}(f$ : $S) \geq j+(n-2) k$.

As such, it suffices to prove the case $i \leq h-1$. If $\alpha=s_{i}$ and $\beta=s_{i+1}$, then $f\left(x_{\alpha+2}\right)<f\left(y_{2}\right) \leq f\left(y_{q-1}\right)<f\left(x_{\beta}\right)$. From Lemma $2, \beta-\alpha \leq n-1$. From Lemma 1, there exist $x \in\left\{x_{\alpha}, x_{\alpha+1}, x_{\alpha+2}\right\}, x^{\prime} \in\left\{x_{\beta}, x_{\beta+1}, x_{\beta+2}\right\}$, $y \in\left\{y_{1}, y_{2}\right\}$, and $y^{\prime} \in\left\{y_{q-1}, y_{q}\right\}$ such that $x$ and $x^{\prime}$ are adjacent to $y$ and $y^{\prime}$, respectively.

Case 2-1. $f(y)<f\left(x_{\alpha+2}\right)$. Since $f\left(y_{2}\right)>f\left(x_{\alpha+2}\right)$, we have $y=y_{1}$. If $p-\alpha \geq n$, then

$$
\begin{aligned}
\operatorname{span}(f: S) & \geq f\left(x_{p}\right)-f\left(y_{1}\right) \\
& =\left(f\left(x_{p}\right)-f\left(x_{\alpha+2}\right)\right)+\left(f\left(x_{\alpha+2}\right)-f\left(y_{1}\right)\right) \geq j+(n-2) k .
\end{aligned}
$$


If $p-\alpha \leq n-1$, then $q+\alpha=(p+q)-(p-\alpha) \geq 2 n+1-(n-1)=n+2$. From Lemma 1, some $x \in\left\{x_{\alpha}, x_{\alpha+1}, x_{\alpha+2}\right\}$ is adjacent to some $\tilde{y} \in\left\{y_{2}, y_{3}\right\}$, and we have $f\left(y_{3}\right)-f\left(x_{\alpha}\right) \geq|f(x)-f(\tilde{y})| \geq j$. Therefore,

$$
\begin{aligned}
\operatorname{span}(f: S) & \geq f\left(y_{q}\right)-f\left(x_{1}\right) \\
& =\left(f\left(y_{q}\right)-f\left(y_{3}\right)\right)+\left(f\left(y_{3}\right)-f\left(x_{\alpha}\right)\right)+\left(f\left(x_{\alpha}\right)-f\left(x_{1}\right)\right) \\
& \geq(q-3) k+j+(\alpha-1) k=j+(q+\alpha-4) k \geq j+(n-2) k .
\end{aligned}
$$

Case 2-2. $f\left(y^{\prime}\right)>f\left(x_{\beta}\right)$. We can show the case $\operatorname{span}(f: S) \geq j+(n-2) k$ using a method similar to that used in Case 2-1.

Case 2-3. $f\left(x_{\alpha+2}\right)<f(y)$ and $f\left(y^{\prime}\right)<f\left(x_{\beta}\right)$. Since $f\left(y^{\prime}\right)-f(y) \geq f\left(y_{q-1}\right)-$ $f\left(y_{2}\right) \geq(q-3) k$, we have

$$
\begin{aligned}
\operatorname{span}(f: S) & \geq f\left(x_{p}\right)-f\left(x_{1}\right)=\left(f\left(x_{p}\right)-f\left(x_{\beta+2}\right)\right)+\left(f\left(x_{\beta+2}\right)-f\left(y_{q-1}\right)\right) \\
& +\left(f\left(y_{q-1}\right)-f\left(y_{2}\right)\right)+\left(f\left(y_{2}\right)-f\left(x_{\alpha}\right)\right)+\left(f\left(x_{\alpha}\right)-f\left(x_{1}\right)\right) \\
& \geq(p-\beta-2) k+j+(q-3) k+j+(\alpha-1) k \\
& =2 j+(p+q+\alpha-\beta-6) k \geq 2 j+(2 n+1-(n-1)-6) k \\
& =2 j+(n-4) k \geq j+(n-2) k .
\end{aligned}
$$

(3) If $p \geq n+2$, then

$$
\operatorname{span}(f: S) \geq f\left(x_{p}\right)-f\left(x_{1}\right) \geq(p-1) k \geq(n+1) k \geq n k+1 .
$$

If $p=n+1$, then $q=n$. From Lemmas 2 and 3, there exists $t$ such that $1 \leq t \leq n-1$ and $\left\{x_{t}, x_{t+1}, x_{t+2}\right\}$ contains a pair of vertices that are set oblique. Then, from Lemma 1 , there exist $h, l$ such that $t \leq h \leq t+2, t \leq l \leq t+1$, and $x_{h}$ is adjacent to $y_{l}$. If $f\left(x_{h}\right)<f\left(y_{l}\right)$ and $f\left(x_{t+2}\right)>f\left(y_{l}\right)$, then

$$
\begin{aligned}
\operatorname{span}(f: S)= & f\left(x_{n+1}\right)-f\left(x_{1}\right)=\left(f\left(x_{n+1}\right)-f\left(x_{t+2}\right)\right) \\
& +\left(f\left(x_{t+2}\right)-f\left(y_{l}\right)\right)+\left(f\left(y_{l}\right)-f\left(x_{h}\right)\right)+\left(f\left(x_{h}\right)-f\left(x_{1}\right)\right) \\
\geq & (n-t-1) k+1+2 k+(h-1) k \geq(n-t+h) k+1 \geq n k+1 .
\end{aligned}
$$

If $f\left(x_{h}\right)<f\left(y_{l}\right)$ and $f\left(x_{t+2}\right)<f\left(y_{l}\right)$, then

$$
\begin{aligned}
\operatorname{span}(f: S)= & \left(f\left(y_{n}\right)-f\left(y_{l}\right)\right) \\
& +\left(f\left(y_{l}\right)-f\left(x_{t+2}\right)\right)+\left(f\left(x_{t+2}\right)-f\left(x_{1}\right)\right) \\
\geq & (n-l) k+1+(t+2-1) k=(n-l+t+1) k+1 \geq n k+1 .
\end{aligned}
$$

If $f\left(x_{h}\right)>f\left(y_{l}\right)$ and $f\left(x_{t}\right)>f\left(y_{l}\right)$, then

$$
\begin{aligned}
\operatorname{span}(f: S)= & f\left(x_{n+1}\right)-f\left(y_{1}\right)=\left(f\left(x_{n+1}\right)-f\left(x_{t}\right)\right)+\left(f\left(x_{t}\right)-f\left(y_{l}\right)\right) \\
& +\left(f\left(y_{l}\right)-f\left(y_{1}\right)\right) \\
\geq & (n+1-t) k+1+(l-1) k=(n-t+l) k+1 \geq n k+1 .
\end{aligned}
$$


If $f\left(x_{h}\right)>f\left(y_{l}\right)$ and $f\left(x_{t}\right)<f\left(y_{l}\right)$, then

$$
\begin{aligned}
\operatorname{span}(f: S)= & \left(f\left(x_{n+1}\right)-f\left(x_{h}\right)\right)+\left(f\left(x_{h}\right)-f\left(y_{l}\right)\right) \\
& +\left(f\left(y_{l}\right)-f\left(x_{t}\right)\right)+\left(f\left(x_{t}\right)-f\left(x_{1}\right)\right) \\
\geq & (n+1-h) k+2 k+1+(t-1) k \\
= & (n-h+t+2) k+1 \geq n k+1 .
\end{aligned}
$$

\section{MAIN THEOREMS}

Theorem 1. If $1 \leq j \leq n+2$, then the distance 3 number $\lambda_{G}(j, 1,1)$ of $G=$ $K_{n} \times K_{m} \times K_{2}(n \geq m \geq 3)$ is $2 m n-1$.

Proof. Since the diameter of $G$ is 3 and $|V|=2 m n, \lambda_{G}(j, 1,1) \geq 2 m n-1$. Let $\tilde{f}: V \rightarrow[0, N]$, and

$$
\tilde{f}\left(u_{0}, u_{1}, u_{2}\right)= \begin{cases}u_{0}+n u_{1}, & \text { if } u_{2}=0 \\ 2 m n-u_{0}-n u_{1}-1, & \text { if } u_{2}=1\end{cases}
$$

Let $u=\left(u_{0}, u_{1}, u_{2}\right)$ and $v=\left(v_{0}, v_{1}, v_{2}\right)$ be distinct vertices of $G$. If $u$ and $v$ are adjacent, since $u_{0} \neq v_{0}$ and $u_{1} \neq v_{1}, u_{0}+v_{0} \leq 2 n-3$ and $u_{1}+v_{1} \leq 2 m-3$. We can assume that $u_{2}=0$ and $v_{2}=1$. Then,

$$
\begin{aligned}
\tilde{f}(v)-\tilde{f}(u)= & 2 m n-v_{0}-n v_{1}-1-u_{0}-n u_{1} \\
& =2 m n-\left(u_{0}+v_{0}\right)-\left(u_{1}+v_{1}\right) n-1 \\
& \geq 2 m n-(2 n-3)-(2 m-3) n-1=n+2 \geq j .
\end{aligned}
$$

If dist $(u, v)=2$, then $u_{2}=v_{2}$. If $u_{2}=v_{2}=0$, since $u_{0} \neq v_{0}$ or $u_{1} \neq v_{1}$,

\begin{tabular}{|c|c|c|c|c|c|c|c|}
\hline & & \multicolumn{2}{|c|}{$u_{2}=0$} & \multicolumn{3}{|c|}{$u_{2}=1$} & \\
\hline 0 & 5 & 10 & 15 & 39 & 34 & 29 & 24 \\
\hline 1 & 6 & 11 & 16 & 38 & 33 & 28 & 23 \\
\hline 2 & 7 & 12 & 17 & 37 & 32 & 27 & 22 \\
\hline 3 & 8 & 13 & 18 & 36 & 31 & 26 & 21 \\
\hline 4 & 9 & 14 & 19 & 35 & 30 & 25 & 20 \\
\hline
\end{tabular}
$\tilde{f}(v)-\tilde{f}(u)=\left(v_{0}-u_{0}\right)+\left(v_{1}-u_{1}\right) n \neq 0$. Similarly, $\tilde{f}(v) \neq \tilde{f}(u)$ when $u_{2}=v_{2}=1$. If $\operatorname{dist}(u, v)=3$, then $u_{2} \neq v_{2}$. We can assume that $u_{2}=0$ and $v_{2}=1$. Since $\tilde{f}(u)=u_{0}+u_{1} n \leq m n-1$ and $\tilde{f}(v)=2 m n-u_{1}-v_{1} n \geq m n, \tilde{f}(v) \neq \tilde{f}(u)$. Hence, $\tilde{f}$ is an $L(j, 1,1)$-labeling for $G$. Thus, $\lambda_{G}(j, 1,1)=2 m n-1$.

Table 1 represents the $L(j, 1,1)$-labeling for $K_{5} \times K_{4} \times K_{2}$ when $1 \leq j \leq 7$. It shows that $\lambda_{G}(j, 1,1)=2 m n-1=39$.

Table 1. $L(j, 1,1)$-labeling when $k=1$ and $1 \leq j \leq 7$ 
In Table 1 , the number located in the $\left(u_{0}+1\right)$-th row and $\left(u_{1}+1\right)$-th column of the box over which $u_{2}$ is indicated, is the labeling of the vertex $\left(u_{0}, u_{1}, u_{2}\right)$ of $K_{5} \times K_{4} \times K_{2}$. For example, the number 31 is labeled to the vertex $(3,1,2)$ of $K_{5} \times K_{4} \times K_{2}$. Other figures remain the same.

Theorem 2. If $2 \leq k \leq j \leq 2 k-1$, then the distance 3 labeling $\lambda_{G}(j, k, 1)$ for $G=K_{n} \times K_{m} \times K_{2}(n \geq m \geq 3)$ is $(m n-1) k+1$.

Proof. Let $f$ be an $L(j, k, 1)$-labeling for $G, V_{0}=\left\{x_{1}, x_{2}, \cdots, x_{m n}\right\}$, and $V_{1}=\left\{y_{1}, y_{2}, \cdots, y_{m n}\right\}$. We can assume that $f\left(x_{i}\right)<f\left(x_{i+1}\right)$ and $f\left(y_{i}\right)<f\left(y_{i+1}\right)$ for all $i=1,2, \cdots, m n-1$, and $f\left(x_{m n}\right)<f\left(y_{m n}\right)$. Then,

$$
\begin{aligned}
\lambda_{G}(j, k, 1) & \geq f\left(y_{m n}\right)-f\left(x_{1}\right)=\left(f\left(y_{m n}\right)-f\left(x_{m n}\right)\right)+\left(f\left(x_{m n}\right)-f\left(x_{m n-1}\right)\right) \\
& +\left(f\left(x_{m n-1}\right)-f\left(x_{m n-2}\right)\right)+\cdots+\left(f\left(x_{2}\right)-f\left(x_{1}\right)\right) \geq 1+(m n-1) k .
\end{aligned}
$$

Let $\tilde{f}: V \rightarrow[0,(m n-1) k+1]$, and

$$
\tilde{f}\left(u_{0}, u_{1}, u_{2}\right)= \begin{cases}u_{0} k+u_{1} n k+u_{2}, & u_{1} \text { is even } \\ \left(n-u_{0}-1\right) k+u_{1} n k+u_{2}, & u_{1} \text { is odd }\end{cases}
$$

Let $u=\left(u_{0}, u_{1}, u_{2}\right)$ and $v=\left(v_{0}, v_{1}, v_{2}\right)$ be distinct vertices of $G$. We can assume that $u_{1} \leq v_{1}$. If $u$ and $v$ are adjacent, since $u_{0} \neq v_{0}$ and $u_{1} \neq v_{1}, u_{0}+v_{0} \leq 2 n-3$ and $u_{1}+v_{1} \leq 2 m-3$. If $v_{1}-u_{1} \geq 2$, since $\tilde{f}(u) \leq u_{1} n k+(n-1) k+1$ and $\tilde{f}(v) \geq v_{1} n k \geq\left(u_{1}+2\right) n k, \tilde{f}(v)-\tilde{f}(u) \geq(n+1) k-1 \geq 2 k-1 \geq j$. If $v_{1}-u_{1}=1$ and $u_{1}$ is even,

$$
\begin{aligned}
\tilde{f}(v)-\tilde{f}(u) & =\left(n-u_{0}-v_{0}-1\right) k+\left(v_{1}-u_{1}\right) n k \\
& \geq(n-(2 n-3)-1) k+n k-1=2 k-1 \geq j .
\end{aligned}
$$

Similarly, we can get that $\tilde{f}(v)-\tilde{f}(u) \geq j$ when $v_{1}-u_{1}=1$ and $u_{1}$ is odd.

If $\operatorname{dist}(u, v)=2$, then $u \neq v$ and $u_{2}=v_{2}$. We can assume that $u_{1} \leq v_{1}$. If $u_{1}<v_{1}$, then

$$
\begin{aligned}
\tilde{f}(v)-\tilde{f}(u) & =v_{1} n k+u_{2}-\left(u_{1} n k+(n-1) k+u_{2}\right) \\
& \geq\left(v_{1}-u_{1}\right) n k-(n-1) k \geq k
\end{aligned}
$$

If $u_{1}=v_{1}$, then

$$
|\tilde{f}(v)-\tilde{f}(u)|=\left|v_{0}-u_{0}\right| k \geq k
$$

If $\operatorname{dist}(u, v)=3$, since $u_{2} \neq v_{2}, \tilde{f}(v) \equiv v_{2} \not \equiv u_{2} \equiv \tilde{f}(u)(\bmod k)$. As such, $\tilde{f}(v) \neq \tilde{f}(u)$. Thus, $\tilde{f}$ is an $L(j, k, 1)$-labeling for $G$. Hence, $\lambda_{G}(j, k, 1) \leq(m n-$ $1) k+1$. 
Table 2 represents the $L(j, k, 1)$-labeling for $K_{5} \times K_{4} \times K_{2}$ when $2 \leq k \leq j \leq$ $2 k-1$. We can see that $\lambda_{G}(j, k, 1)=(m n-1) k+1=19 k+1$.

Table 2. $L(j, k, 1)$-labeling when $2 \leq k \leq j \leq 2 k-1$

$\left.\begin{array}{|cccc}\multicolumn{8}{c}{u_{2}=0} \\ \hline 0 & 9 k & 10 k & 19 k \\ k & 8 k & 11 k & 18 k \\ 2 k & 7 k & 12 k & 17 k \\ 3 k & 6 k & 13 k & 16 k \\ 4 k & 5 k & 14 k & 15 k\end{array}\right]\left[\begin{array}{cccc|}1 & 9 k+1 & 10 k+1 & 19 k+1 \\ k+1 & 8 k+1 & 11 k+1 & 18 k+1 \\ 2 k+1 & 7 k+1 & 12 k+1 & 17 k+1 \\ 3 k+1 & 6 k+1 & 13 k+1 & 16 k+1 \\ 4 k+1 & 5 k+1 & 14 k+1 & 15 k+1 \\ \hline\end{array}\right.$

Corollary 1. The radio number $\operatorname{rn}(G)=\lambda_{G}(3,2,1)$ for $G=K_{n} \times K_{m} \times K_{2}(n \geq$ $m \geq 3)$ is $2 m n-1$.

Table 3 represents the radio number for $K_{5} \times K_{4} \times K_{2}$. We can see that $\mathrm{rn}(\mathrm{G})=39$.

Table 3. Radio labeling

\begin{tabular}{|c|c|c|c|c|c|c|c|}
\hline & & \multicolumn{2}{|c|}{$u_{2}=0$} & \multicolumn{3}{|c|}{$u_{2}=1$} & \\
\hline 0 & 18 & & 38 & 1 & & 21 & 39 \\
\hline 2 & 16 & 22 & 36 & 3 & 17 & 23 & 37 \\
\hline 4 & 14 & 24 & 34 & 5 & 15 & 25 & 35 \\
\hline 6 & 12 & 26 & 32 & 7 & 13 & 27 & 33 \\
\hline 8 & 10 & 28 & 30 & 9 & 11 & 29 & 31 \\
\hline
\end{tabular}

Theorem 3. If $k \geq 2$ and $j=2 k$, then the distance 3 labeling $\lambda_{G}(j, k, 1)$ for $G=K_{n} \times K_{m} \times K_{2}(n \geq m \geq 3)$ is $(m n-1) k+m$.

Proof. Let $f$ be an $L(j, k, 1)$-labeling for $G$. If $S=\left\{x_{1}, x_{2}, \cdots, x_{2 m n}\right\}$ and $f\left(x_{1}\right)<f\left(x_{2}\right)<\cdots<f\left(x_{2 m n}\right)$, from Proposition 2 (3),

$$
\begin{aligned}
\operatorname{span}(f)= & f\left(x_{2 m n}\right)-f\left(x_{1}\right) \\
= & \left(f\left(x_{2 m n}\right)-f\left(x_{2 m n-1}\right)\right)+\left(f\left(x_{2 m n-1}\right)-f\left(x_{2 m n-3}\right)\right) \\
& +\left(f\left(x_{2 m n-3}\right)-f\left(x_{2 m n-5}\right)\right)+\cdots+\left(f\left(x_{2 m n-2 n+3}\right)-f\left(x_{2 m n-2 n+1}\right)\right) \\
& +\left(f\left(x_{2 m n-2 n+1}\right)-f\left(x_{2 m n-4 n+1}\right)\right) \\
& +\left(f\left(x_{2 m n-4 n+1}\right)-f\left(x_{2 m n-6 n+1}\right)\right)+\cdots+\left(f\left(x_{2 n+1}\right)-f\left(x_{1}\right)\right) \\
\geq 1 & +(n-1) k+(n k+1)(m-1)=(m n-1) k+m .
\end{aligned}
$$

Let $\tilde{f}: V \rightarrow[0,(m n-1) k+m]$, and

$$
\tilde{f}\left(u_{0}, u_{1}, u_{2}\right)= \begin{cases}u_{0} k+(n k+1) u_{1}+u_{2}, & u_{1} \text { is even } \\ \left(n-u_{0}-1\right) k+(n k+1) u_{1}+u_{2}, & u_{1} \text { is odd. }\end{cases}
$$


Then, using a method similar to that used in Theorems 1 and 2, we get that $\tilde{f}$ is an $L(j, k, 1)$-labeling for $G$. Hence, $\lambda_{G}(j, k, 1)=(m n-1) k+m$.

Table 4 represents an $L(j, k, 1)$-labeling for $K_{5} \times K_{4} \times K_{2}$ when $k \geq 2$ and $j=2 k$. We can see that $\lambda_{G}(j, k, 1)=(m n-1) k+m=19 k+4$.

Table 4. $L(j, k, 1)$-labeling when $k \geq 2$ and $j=2 k$

\begin{tabular}{|cccc}
\hline 0 & $9 k+1$ & $10 k+2$ & $19 k+3$ \\
$k$ & $8 k+1$ & $11 k+2$ & $18 k+3$ \\
$2 k$ & $7 k+1$ & $12 k+2$ & $17 k+3$ \\
$3 k$ & $6 k+1$ & $13 k+2$ & $16 k+3$ \\
$4 k$ & $5 k+1$ & $14 k+2$ & $15 k+3$
\end{tabular} \mid \begin{tabular}{ccccc|}
1 & $9 k+2$ & $10 k+3$ & $19 k+4$ \\
$k+1$ & $8 k+2$ & $11 k+3$ & $18 k+4$ \\
$2 k+1$ & $7 k+2$ & $12 k+3$ & $17 k+4$ \\
$3 k+1$ & $6 k+2$ & $13 k+3$ & $16 k+4$ \\
$4 k+1$ & $5 k+2$ & $14 k+3$ & $15 k+4$ \\
\hline
\end{tabular}

Theorem 4. If $k \geq 2$ and $2 k+1 \leq j \leq n k$, then for $G=K_{n} \times K_{m} \times K_{2}(n \geq$ $m \geq 3)$ we have $\lambda_{G}(j, k, 1)=(m-1) j+(m n-2 m+1) k+1$.

Proof. Let $f$ be an $L(j, k, 1)$-labeling for $G$ and $a_{1}, a_{2}, \cdots, a_{2 m n}$ be the rearrangement of all elements of $V$ such that $f\left(a_{1}\right)<f\left(a_{2}\right)<\cdots<f\left(a_{2 m n}\right)$. Since $j \leq n k, j+(n-2) k \leq(2 n-2) k$. Hence, from Proposition 1, $f\left(a_{2 n(i+1)+1}\right)-$ $f\left(a_{2 n i+1}\right) \geq j+(n-2) k$ for all $i=0,1, \cdots, m-1$. Thus,

$$
\begin{aligned}
\operatorname{span}(f) & =f\left(a_{2 m n}\right)-f\left(a_{1}\right)=\left(f\left(a_{2 m n}\right)-f\left(a_{2 m n-1}\right)\right) \\
& +\left(f\left(a_{2 m n-1}\right)-f\left(a_{2 m n-3}\right)\right)+\left(f\left(a_{2 m n-3}\right)-f\left(a_{2 m n-5}\right)\right)+\cdots \\
& +\left(f\left(a_{2 m n-2 n+3}\right)-f\left(a_{2 m n-2 n+1}\right)\right)+\left(f\left(a_{2 m n-2 n+1}\right)-f\left(a_{2 m n-4 n+1}\right)\right) \\
& +\left(f\left(a_{2 m n-4 n+1}\right)-f\left(a_{2 m n-6 n+1}\right)\right)+\cdots+\left(f\left(a_{2 n+1}\right)-f\left(a_{1}\right)\right) \\
& \geq 1+(n-1) k+(m-1)(j+(n-2) k) \\
& =(m-1) j+(n-1+m n-2 m-n+2) k+1 \\
& =(m-1) j+(m n-2 m+1) k+1 .
\end{aligned}
$$

Let $\tilde{f}: V \rightarrow[0, N]$, and

$$
\tilde{f}\left(u_{0}, u_{1}, u_{2}\right)= \begin{cases}u_{0} k+(j+(n-2) k) u_{1}+u_{2}, & u_{1} \text { is even } \\ \left(n-u_{0}-1\right) k+(j+(n-2) k) u_{1}+u_{2}, & u_{1} \text { is odd, }\end{cases}
$$

where $N=(m-1) j+(m n-2 m+1) k+1$. Then, as in the previous theorems, we can show that $\tilde{f}$ is an $L(j, k, 1)$-labeling for $G$. As such, $\lambda_{G}(j, k, 1) \leq(m-1) j+$ $(m n-2 m+1) k+1$.

Table 5 represents an $L(j, k, 1)$-labeling for $K_{5} \times K_{4} \times K_{2}$ when $k \leq 2$ and $2 k+1 \leq j \leq n k$. We can show that $\lambda_{G}(j, k, 1)=(m-1) j+(m n-2 m+1) k+1=$ $3 j+13 k+1$. 
Table 5. $L(j, k, 1)$-labeling when $k \geq 2$ and $2 k+1 \leq j \leq n k$

$\left.\begin{array}{|cccc}\multicolumn{1}{c}{u_{2}=0} \\
\hline 0 & j+7 k+1 & 2 j+6 k & 3 j+13 k+1 \\
k & j+6 k+1 & 2 j+7 k & 3 j+12 k+1 \\
2 k & j+5 k+1 & 2 j+8 k & 3 j+11 k+1 \\
3 k & j+4 k+1 & 2 j+9 k & 3 j+10 k+1 \\
4 k & j+3 k+1 & 2 j+10 k & 3 j+9 k+1\end{array}\right] \quad$\begin{tabular}{cccc|}
1 & $j+7 k$ & $2 j+6 k+1$ & $3 j+13 k$ \\
$k+1$ & $j+6 k$ & $2 j+7 k+1$ & $3 j+12 k$ \\
$2 k+1$ & $j+5 k$ & $2 j+8 k+1$ & $3 j+11 k$ \\
$3 k+1$ & $j+4 k$ & $2 j+9 k+1$ & $3 j+10 k$ \\
$4 k+1$ & $j+3 k$ & $2 j+10 k+1$ & $3 j+9 k$ \\
\hline
\end{tabular}

\section{ACKNOWLEDGMENTS}

We would like to thank anonymous referee for valuable comments and corrections.

\section{REFERENCES}

1. A. A. Bertossi and M. C. Pinotti, Mappings for conflict-free access of paths in bidimensional arrays, circular lists and complete trees, J. Parallel and Distributed Computing, 62 (2002), 1314-1333.

2. A. A. Bertossi and M. C. Pinotti, Channel assignment with separation for interference avoidance in wireless networks, IEEE Trans. Parallel Distrib. Syst., 14 (2003), 222-235.

3. A. A. Bertossi and M. C. Pinotti, Approximate $L\left(\delta_{1}, \delta_{2}, \cdots, \delta_{t}\right)$-coloring of trees and interval graphs, Networks, 49 (2007), 204-216.

4. A. A. Bertossi, M. C. Pinotti and R. Tan, Efficient use of radio spectrum in weireless networks with channel separation between close stations, DIAL-M 2000 4th International Workshop on Discrete Algorithms and Methods for Mobile Computing and Computations, 2000.

5. T. Calamoneri, E. G. Fusco, R. B. Tan and P. Vocca, $L(h, 1,1)$-labeling of outerplanar graphs, Math. Methods Oper. Res., 69 (2009), 307-321.

6. M. L. Chia, D. Kuo, H. Liao, C. H. Yang and R. K. Yeh, $L(3,2,1)$ labeling of graphs, Taiwanese J. Math., 15 (2011), 2439-2457.

7. J. Fiala, P. Golovach, J. Kratochvil, B. Lidický and D. Paulusma, Distance three labelings of trees, Discrete Applied Math., 160(6) (2012), 764-779.

8. J. R. Griggs and R. K. Yeh, Labeling graphs with a condition at distance two, SIAM J. Disc. Math., 5 (1992), 586-595.

9. W. K. Hale, Frequency assignment: theory and application, Proc. IEEE, 68 (1980), $1497-1514$.

10. E. Haque and P. K. Jha, $L(j, k)$-labelings of Kronecker products of complete graphs, IEEE Trans. on Cir. \& Sys. II, 55 (2008), 70-73.

11. D. S. Kim, D. Z. Du and P. M. Pardalos, A coloring problem on the $n$-cable, Discrete Applied Math., 103 (2000), 307-311. 
12. D. King, C. J. Ras and S. Zhou, The $L(h, 1,1)$-labeling problem for trees, European J. Combinatorics, 31 (2010), 1295-1306.

13. D. D. Liu, Radio number for trees, Discrete Math., 308 (2008), 1153-1164.

14. P. Lam, W. Lin and J. Wu, $L(j, k)$-labelings for the products of complete graphs, $J$. Comb. Optimm., 14 (2007), 219-227.

15. X. Li, V. Mak and S. Zhou, Optimal radio labelings of complete m-ary trees, Discrete Applied Math., 158 (2010), 507-515.

16. D. D. Liu and X. Zhu, Multilevel distance labelings for paths and cycles, SIAM J. Disc. Math., 19 (2005), 610-621.

17. D. D. Liu and M. Xie, Radio number for square paths, Ars. Combinatoria, 90 (2009), 307-319.

18. A. Sen, T. Roxbrorough and S. Medidi, Upper and lower bounds of a class of channel assignment problems in circular networks, Proc. of IEEE INFOCOM '98, 3 (1998), 1284-1291.

19. S. Zhou, A distance-labelling problem for hypercubes, Discrete Applied Math., 156 (2008), 2846-2854.

Byeong Moon Kim and Byung Chul Song

Department of Mathematics

Gangneung-Wonju National University

Gangneung 210-702

Korea

E-mail: kbm@gwnu.ac.kr bcsong@gwnu.ac.kr

Woonjae Hwang

Department of Information and Mathematics

Korea University

Jochiwon 339-700

Korea

E-mail: woonjae@korea.ac.kr 